\title{
Befunderhebung nach Handverletzungen - Klinische Untersuchung und radiologische Diagnostik
}

\author{
Michael Wittemann, Berthold Bickert, Günter Germann
}

\section{Zusammenfassung}

Die Anamnese einer Handverletzung lässt wenig Rückschlüsse auf die Verletzungsschwere $\mathrm{zu}$, ist dennoch hinsichtlich der Latenz zwischen Unfallzeitpunkt und Versorgung wichtig. Tetanusimpfschutz und Tollwutrisiko müssen abgeklärt werden. Die klinische Untersuchung muss einem festen Schema folgen, das die gesamte obere Extremität beinhaltet. Weichteilschäden, Schmerzpunkte, aktive und passive Beweglichkeit, Instabilitäten sowie Störungen von Durchblutung und Sensibilität sind zu beachten. Eine Nativröntgendiagnostik ist obligat. Die verletzte Struktur muss exakt in zwei

\section{Einleitung}

Handverletzungen machen im traumatologischen Alltag einen nicht unerheblichen Prozentsatz des Patientenaufkommens aus (ca. 35\% bei Arbeitsunfällen). Da derzeit nur ein kleiner Teil der Verletzten bereits primär zu einem Handchirurgen oder einem handchirurgischen Zentrum gelangt, obliegen im Allgemeinen die Erstdiagnostik und Erstversorgung chirurgischen und orthopädischen Praxen oder Krankenhäusern der Grundbzw. Grund- und Regelversorgung.

Im Medizinstudium und der chirurgischen Facharztausbildung wird bis dato der Handchirurgie, die man ohne Übertreibung als eigenes Fachgebiet bezeich-

OP-JOURNAL 2001; 17: 216-220

(c) Georg Thieme Verlag Stuttgart · New York
Ebenen dargestellt werden. Bei carpalen Frakturen ist in Einzelfällen die Verletzung nicht auf dem Bild am Unfalltag zu erkennen. Die Computertomographie stellt eine wertvolle Ergänzung des Nativröntgens - insbesondere bei Frakturen im Handgelenksbereich - dar. Sonographie, Röntgendurchleuchtung und Kernspintomographie haben in der Diagnostik nur begrenzte Anwendungsgebiete, können jedoch in Einzelfällen eine wertvolle Hilfe darstellen. Die exakte Dokumentation von Anamnese, Befund und Behandlung ist unerlässlich. Eine Fotodokumentation von Handverletzungen ist in jedem Fall wünschenswert.
Klinische Diagnostik

\section{Anamnese}

Die Anamnese ist oftmals nur bruchstückhaft zu erheben und zu verwerten, da das Entstehen einer Handverletzung im Allgemeinen durch eine sehr schnell einwirkende äußere Gewalteinwirkung bedingt ist und viele Patienten nicht genau sagen können, was im einzelnen wie geschehen ist.

Dennoch muss die Anamnese den Unfallzeitpunkt, den Unfallmechanismus und die Erstbehandlung (Medikamentengabe!) zwischen Unfall und Ankunft in Praxis oder Krankenhaus beinhalten.

Bei offenen Verletzungen ist selbstverständlich der Tetanusimpfschutz des Patienten, bei Tierbissverletzungen auch das Risiko einer Tollwutinfektion abzuklären.

\section{Klinische Untersuchung}

nen kann, noch nicht die Bedeutung beigemessen, die ihr eigentlich zusteht, obwohl in den letzten 10-15 Jahren das Verständnis der Komplexität von Verletzungen und die Operations- und Nachbehandlungsverfahren drastisch verbessert werden konnten.

Daraus resultiert, dass bedingt durch mangelhafte Kenntnisse und Erfahrung der Erstbehandler bei der Diagnostik und Therapie nicht selten Irrtümer und Versäumnisse auftreten, die im weiteren Behandlungsverlauf oftmals nicht wieder gutgemacht werden können und so für die Patienten irreparable Funktionseinbußen mit sich bringen und dann auch - was gerade in der heutigen Zeit zunehmend an Bedeutung gewinnt - für die Kostenträger finanzielle Mehraufwendungen in Millionenhöhe bedingen [7].
Um eine hinreichend aussagekräftige klinische Untersuchung durchführen zu können, ist es erforderlich, dass der Untersucher mit dem Verletzten kommunizieren kann.

Da eine isolierte Handverletzung - auch eine Amputationsverletzung der ganzen Hand - im Allgemeinen nicht zu einer vitalen Gefährdung des Patienten führt, ist eine Intubation am Unfallort oder auf dem Transport nicht indiziert.

Bei der Gabe von Schmerzmitteln ist darauf zu achten, dass diese die Kommunikation des Verletzten mit dem Handchirurgen nicht beeinträchtigen.

Die Gabe von Schmerzmitteln durch den Notarzt sollte intravenös erfolgen, eine orale Gabe ist wegen der Nüchternheit bei einer eventuell erforderlichen Narkose kontraindiziert. 


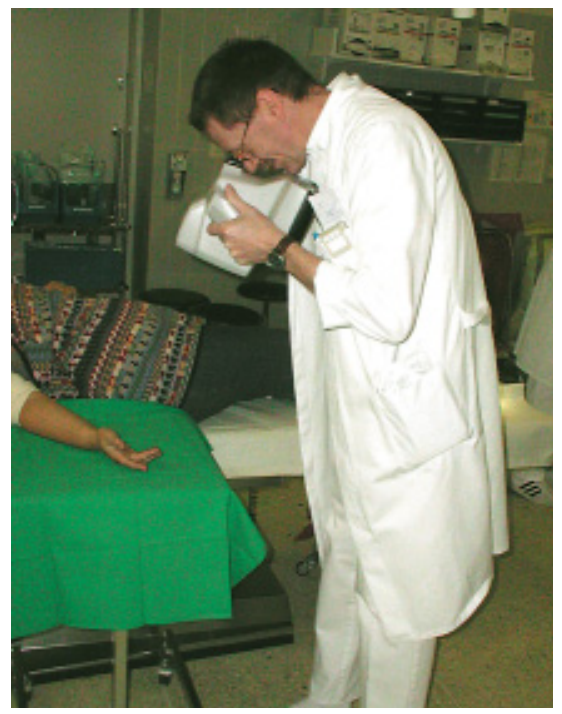

Abb.1 Fotodokumentation einer Handverletzung mittels Sofortbildkamera.

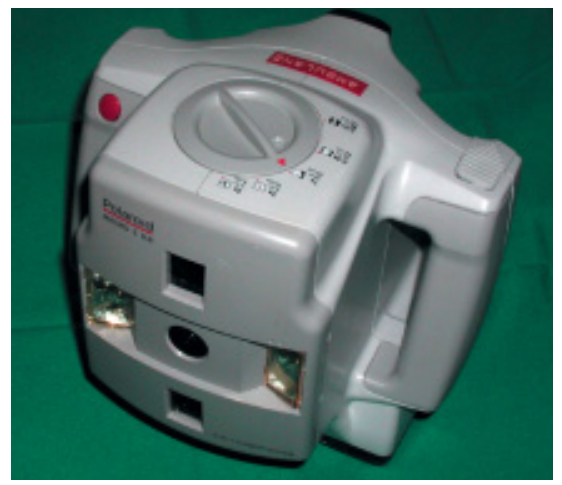

Abb. 2 Sofortbildkamera mit 5 einstellbaren Brennweiten (Polaroid 5 SLR).

Auf eine Lokal- oder Leitungsanästhesie muss bis zur Ankunft des Verletzten an der entgültigen Versorgungsstätte verzichtet werden, da dies natürlich die Überprüfung und Dokumentation der Sensibilität unmöglich macht!

Bei der klinischen Diagnostik ist es wichtig, dass der Untersucher einen standardisierten Untersuchungsablauf der gesamten oberen Extremität durchführt, von dem er sich auch durch offensichtliche Verletzungen nicht abbringen lässt.

Bei offenen Verletzungen wird zunächst unter sterilen Bedingungen der Verband geöffnet und vorsichtig - soweit es der Verletzte toleriert - die Extremität mit einer alkoholfreien Desinfektionslösung von Blut und Schmutz gereinigt, um sich einen Überblick über das Verletzungsausmaß verschaffen zu können. Blutung und Verschmutzung täuschen oft ein deutlich größeres Verletzungsausmaß vor, als in Wirklichkeit vorliegt.

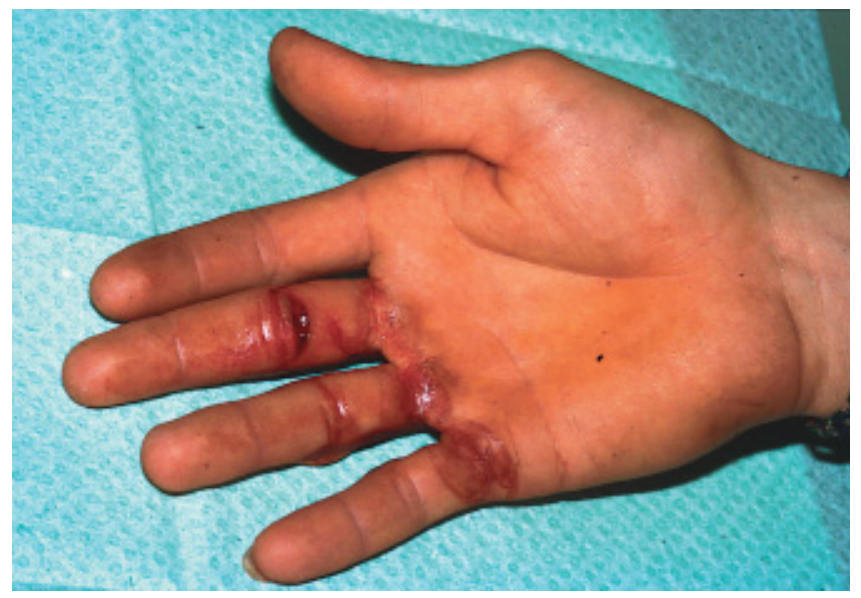

Abb. 3 Kleine Schnittwunde am Mittelfinger.

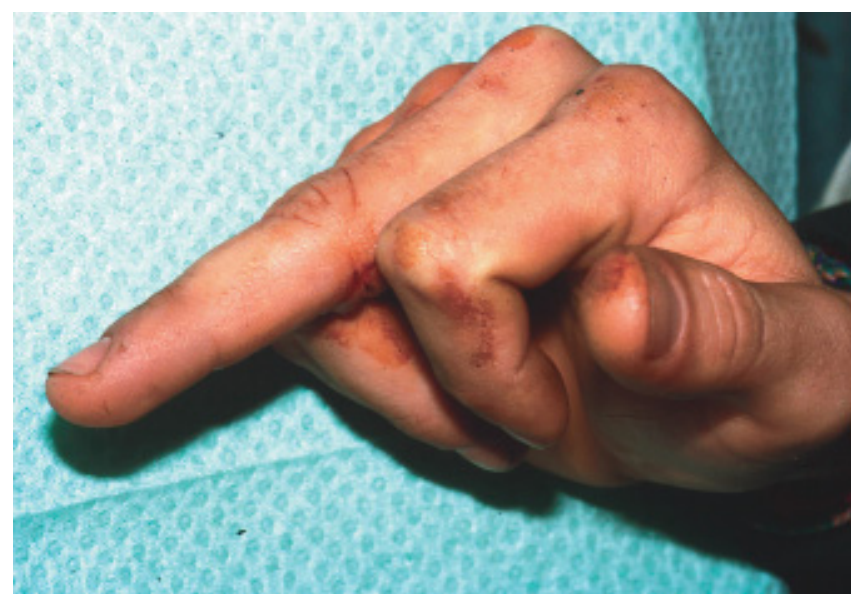

Abb. 4 Finger in Mittel- und Endgelenk nicht aktiv beugbar - hochgradiger V.a. Verletzung beider Beugesehnen, intraoperativ bestätigt.

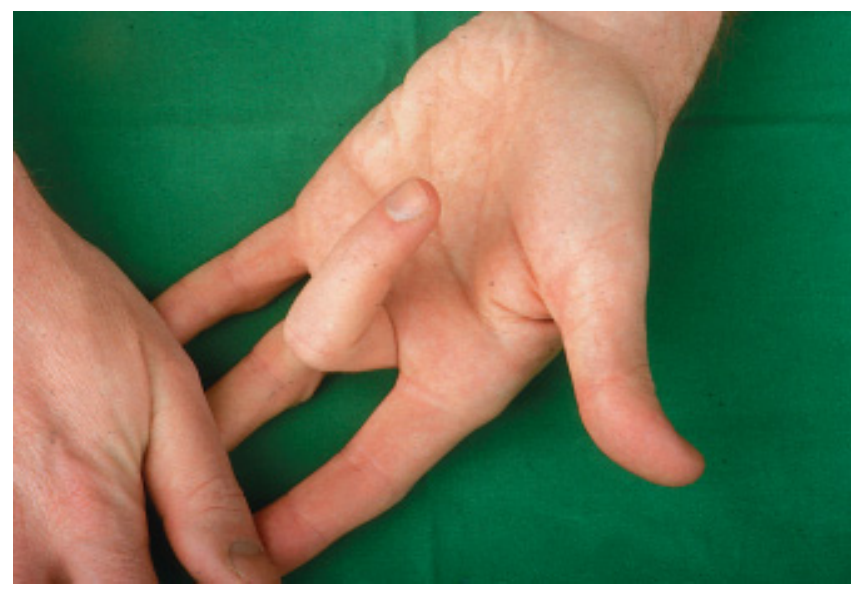

Abb. 5 klinische Prüfung des oberflächlichen Langfingerbeugers.

Nach der Reinigung erfolgt die Inspektion der verletzten Hand um Weichteilschäden, Schwellungen und Fehlstellung erkennen und dokumentieren zu können.

$\mathrm{Zu}$ diesem Zeitpunkt sollte obligat eine Fotodokumentation der Verletzung sowohl in Übersichts- als auch in Detailaufnahmen erfolgen, was am besten mit einer Polaroid- oder Digitalkamera durchgeführt werden kann (Abb.1 u.2). Die Aufnahmen müssen mit den Personalien des Unfallverletzten und dem Zeit- punkt der Dokumentation versehen werden.

Nach der Inspektion wird eine Prüfung der aktiven Beweglichkeit vorgenommen (Abb. 3 u. 4).

Diese beinhaltet die Beweglichkeit aller Gelenke von Ellenbogengelenk abwärts sowie die Funktionsprüfung aller Sehnen, wobei an den Langfingern tiefe und oberflächliche Beugesehnenfunktion isoliert zu überprüfen sind (Abb.4 u.5). 


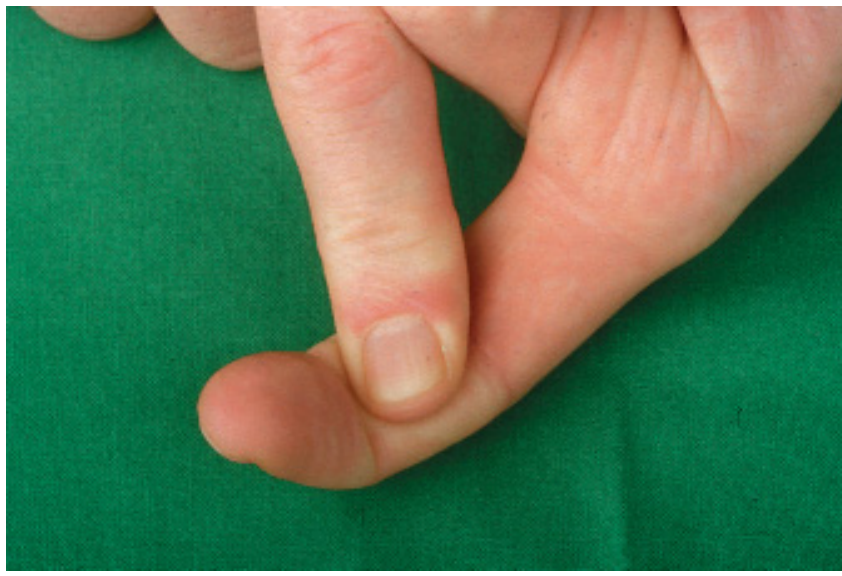

Abb. 6 Klinische Prüfung des tiefen Langfingerbeugers.

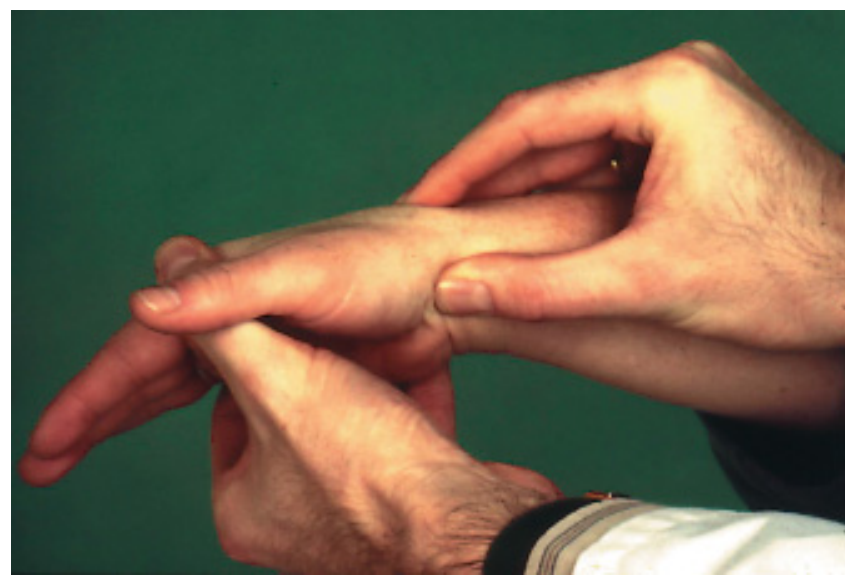

Abb. 7 Watson-Test: Der Daumen des Untersuchers drückt von palmar gegen das Tuberculum scaphoidei. Bei Radialduktion des Handgelenks lässt sich die Palmarflexion des Kahnbeins bei intaktem scapholunären Band auch durch starken Druck nicht verhindern, bei Verletzung des SL-Bands lässt sich jedoch das Kahnbein durch den Daumendruck des Untersuchers schmerzhaft nach dorsal subluxieren.
Der nächste Untersuchungsschritt ist Palpation. Hierbei werden Ödeme, Hämatome, Gelenkergüsse und Schmerzpunkte festgestellt.

Nach der Palpation wird die Prüfung der passiven Gelenkbeweglichkeit und der Bandstabilität durchgeführt. Der Untersucher muss bei diesem Schritt äußerste Vorsicht walten lassen, da er dem Patienten teilweise starke Schmerzen zufügen kann, was in reflektorischen Muskelkontraktionen resultiert, die das Untersuchungsergebnis dann letztlich verfälschen und insbesondere bei Bandverletzungen auch zum Nichterkennen von Instabilitäten führt.

Die Prüfung der Bandstabilität ist insbesondere bei Handgelenksverletzungen (Watson-Test, ulnarer-Stress-Test, FoveaZeichen, Ballotment) $[4,6]$ durch das bereits kurz nach dem Unfall auftretende Gelenkhämatom zunächst oftmals unsicher, so dass diese Untersuchungen häufig nach einer Immobilisation von 5-8 Tagen und einem Rückgang des Hämatoms wesentlich aussagekräftiger sind (Abb. 7).

Weiterhin erfordert die klinische Prüfung des karpalen Bandapparats vom Untersucher eine großes Maß an Erfahrung um pathologische Befunde richtig zu werten und auch nicht zu überschätzen [2].

Der letzte Schritt der klinischen Untersuchung einer verletzten Hand ist die Überprüfung von Durchblutung und Sensibilität.
Eine arterielle Verletzung resultiert nicht zwangsläufig in einer aufgehobenen Durchblutung der Hand oder eines Teils davon.

Bedingt durch die doppelte (bei Vorliegen einer kräftigen $A$. mediana sogar dreifachen) arterielle Versorgung der Hand und der vierfachen ( 2 palmare und 2 dünnere dorsale Arterien) arteriellen Versorgung der Finger kann eine isolierte Arterienverletzung im allgemeinen bei gesunden Gefäßen problemlos kompensiert werden. Hier ist der Allen-Test ein wertvolles klinisches Hilfsmittel zur Erkennung der Verletzung.

Beim Allen-Test wird die unverletzte kontralaterale Arterie manuell komprimiert, was dann bei Vorliegen einer Arterienverletzung zu einer deutlichen Verlangsamung oder Aufhebung der Rekapillarisierung der Hand oder des Fingers führt und nach Beendigung der Kompression die Rekapillarisierung wieder normalisiert.

Bei der Prüfung der Sensibilität ist es wichtig zu wissen, dass Patienten nach vollständiger Durchtrennung eines Stamm- oder Fingernerven manchmal noch mehrere Stunden nach dem Unfall im Versorgungsgebiet des verletzten Nerven eine normale Sensibilität angeben können. Dies bedeutet, dass eine normale Sensibilitätsangabe eine Nervenverletzung letztlich nicht ausschließt.

Da trophische Störungen naturgemäß kurze Zeit nach einem Unfall noch nicht vorliegen, ist die Überprüfung der Trophik nach einer frischen oder kurz zu- rückliegenden Handverletzung nicht richtungweisend.

Dennoch ist bei der klinischen Untersuchung nach einer Handverletzung natürlich auch auf Folgen früherer Erkrankungen, Verletzungen oder Fehlbildungen sowie auf zusätzliche unfallunabhängige Veränderungen (z.B. Dupuytrensche Kontraktur) zu achten.

\section{Apparative Diagnostik}

\section{Nativröntgen}

Die Anfertigung von Röntgenaufnahmen in zwei Ebenen ist bei jeder Handverletzung, bei der eine mechanische Gewalteinwirkung vorliegt, unverzichtbar.

Dies bedeutet, dass beispielsweise auch bei einer klinisch primär harmlos imponierenden Schnittverletzung geröngt werden muss, um vorhandene Fremdkörper oder Gelenksubluxationen bei Durchtrennung von Bändern verifizieren $\mathrm{zu}$ können (Abb.8a u.b). Ebenso kann eine Röntgenaufnahme Folgen von vorausgegangenen Verletzungen oder präexistente degenerative Veränderungen aufdecken.

Die Röntgenuntersuchung erfolgt selbstverständlich erst nach der klinischen Untersuchung, damit sie einerseits zielgerichtet das verletzte Areal darstellt und andererseits nicht zum Übersehen von weiteren Veränderungen führt. 


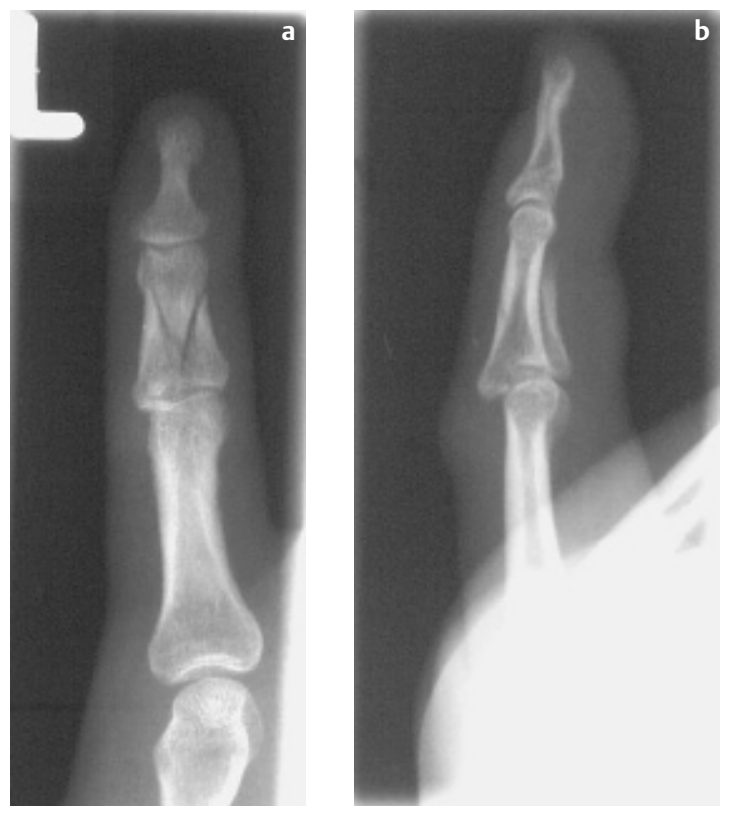

Abb. 8a u.b Mittelgliedbasisfraktur eines Kleinfingers: Die Schädigung der Gelenkfläche und die Subluxation des Mittelgelenks lässt sich nur auf der seitlichen Aufnahme in vollem Ausmaß beurteilen.
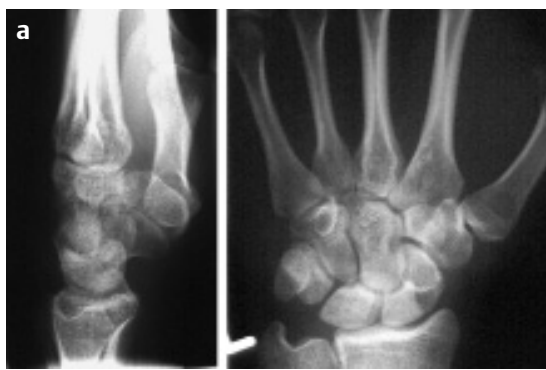

b

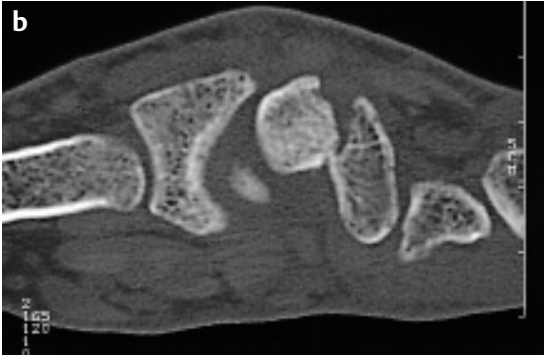

Abb.10au.b Kahnbeinbruch: im Nativröntgenbild undisloziert erscheinend, im CT deutliche Dislokation, d.h. OP-Indikation.

\section{Computertomographie (CT)}

eine vorliegende Fraktur, so dass oft erst die Wiederholung der Röntgenaufnahme nach einer Woche den Schaden aufdeckt.

\section{Röntgendurchleuchtung}

Die Durchleuchtung ist aufgrund der relativ hohen Strahlenbelastung, der Patient und Untersucher ausgesetzt werden, nicht zu den radiologischen Verfahren der ersten Wahl zu rechnen.

Bei Verletzungen des Handgelenks sind häufig Spezialaufnahmen $\left(20^{\circ}\right.$ ulnar angehoben, Stecher-Aufnahme, Karpaltunnelaufnahme etc.) erforderlich, um eine genauere Beurteilung von einzelnen Knochen oder Gelenken zu ermöglichen, wobei bei der Röntgendiagnostik von karpalen Instabilitäten immer eine Vergleichsaufnahme der unverletzten Gegenseite mitgemacht werden sollte.

Die Nativradiologie zeigt bei Verletzungen der Handwurzelknochen, insbeson-

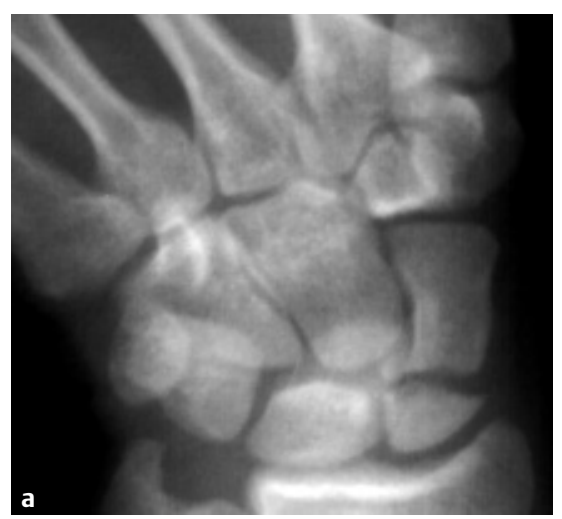

Sie kann jedoch bei Instabilitäten nach Bandverletzungen des Handgelenks oder bei primär nicht sicher klassifizierbaren Frakturen ein wertvolles Hilfsmittel zur Therapieplanung darstellen (Abb.9a-c).

Der Einsatz der Röntgendurchleuchtung muss durch einen erfahrenen Untersucher indiziert werden, bei der Durchführung ist auf eine möglichst kurze Durchleuchtungszeit zu achten.

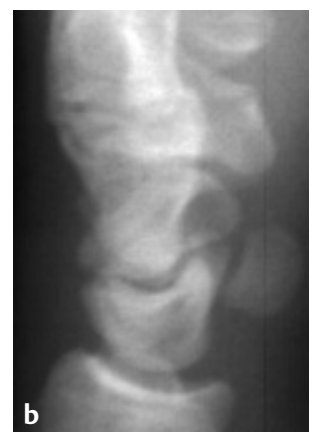

Die hochauflösende Computertomographie hat in den letzten Jahren eine deutliche Verfeinerung der Diagnostik von Verletzungen oder Verletzungsfolgen vor allem des Handgelenks ermöglicht.

Gerade beim Kahnbeinbruch, aber auch bei anderen Frakturen und Gelenkverletzungen an der Hand ist durch die CT eine wesentlich differenziertere Indikationsstellung für operative oder nicht operative Verfahren möglich [1].

In unserer Klinik wird beispielsweise die Indikation zur konservativen Behandlung des Kahnbeinbruchs nur dann gestellt, wenn durch CT nachgewiesen ist, dass keine Fehlstellung der Fraktur vorliegt (Abb.10a u.b).

Weiterhin sind posttraumatische Arthrosen und Fehlstellungen durch die CT häufig besser beurteilbar. tionsfraktur: in Nativröntgenbild „nur“ als Kahnbeinbruch imponierend. Unter Durchleuchtung ist das volle Ausmaß der Instabilität deutlich. 
Es ist jedoch wichtig, dass bei Anfertigung eines Computertomogramms der Handchirurg dem Radiologen genau mitteilt in welcher Ebene die Schichten verlaufen sollen, da nur exakt in der Knochen- oder Gelenkachse liegende Schnitte eine hinreichend genaue Aussage ermöglichen.

\section{Kernspintomographie (MRT)}

Der Anwendung der Kernspintomographie ist bei Handverletzungen begrenzt.

Sie kann in Einzelfällen durchgeführt werden, wenn durch Darstellung des Frakturödems eine möglichst schnelle Diagnosesicherung bei negativem Ergebnis von Nativröntgen oder CT erfolgen soll (Abb.11 a-c).

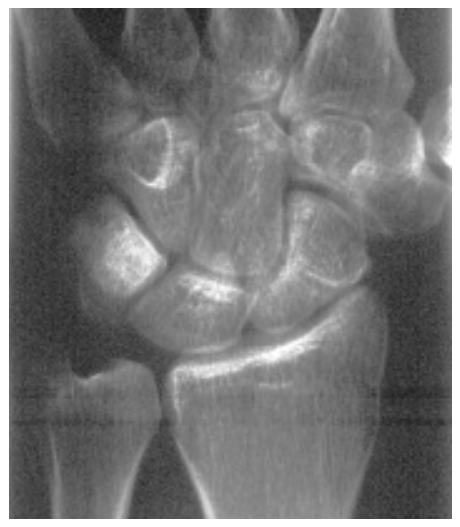

a
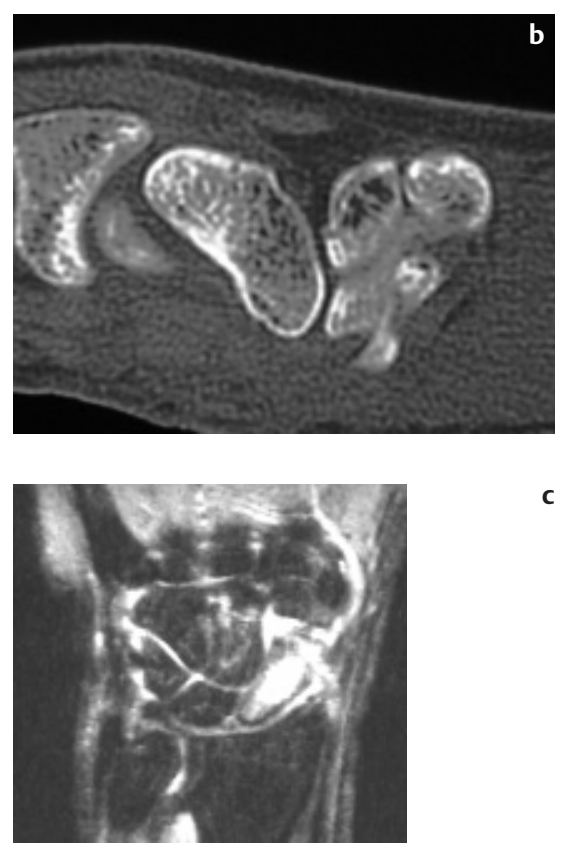

Abb.11a-c Kahnbeinbruch: im Nativröntgenbild und im CT keine Fraktur sichtbar, im MRT deutliches Knochenmarködem als Frakturhinweis.
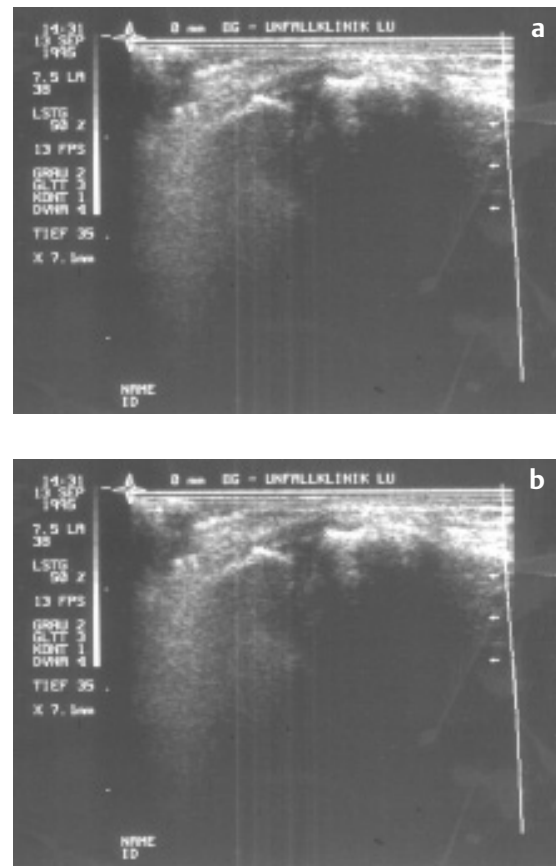

Abb.12au.b ulnare Seitenbandruptur am Daumengrundgelenk: deutliches Klaffen des Gelenks unter Stress.

In der Diagnostik von Band- oder Diskusverletzungen des Handgelenks hat die MRT keine Indikation, da sie einerseits keine ausreichende Genauigkeit aufweist und andererseits mit der Arthroskopie eine deutlich genauere, wenn auch invasivere Methode zur Verfügung steht [3].

Die MRT stellt jedoch das Verfahren der ersten Wahl dar, wenn im weiteren Verlauf nach einer Handverletzung eine Aussage über die Vitalität von Knochenanteilen erforderlich ist, wie es beispielsweise bei Kahnbeinpseudarthrosen mit kleinem proximalen Fragment oder der Lunatumnekrose der Fall ist.

In diesen Fällen muss die MRT jedoch unbedingt mit der Gabe von GadolinumKontrastmittel durchgeführt werden.

\section{Sonographie}

Die hochauflösende Sonographie mit Schallköpfen mit Frequenzen von $7,5 \mathrm{MHz}$ und mehr hat bisher in der Handchirurgie keine weite Verbreitung gefunden.

Dies ist bedingt durch die Tatsache, dass mit der Sonographie nur Weichteilstrukturen genau dargestellt werden können, deren Schäden und Funktionsstörungen im Allgemeinen auch klinisch mit hinreichender Sicherheit zu beurteilen sind.

Dennoch kann die Sonographie bei der Diagnostik von Sehnenrupturen, Gelenk- instabilitäten an den Fingern - insbesondere bei der Ruptur des ulnaren Seitenbands am Daumengrundgelenk (Abb.12au.b) - sowie der Differenzierung von Weichteilschwellungen und Tumoren einen wichtigen diagnostischen Beitrag liefern [5].

\section{Dokumentation}

Eine exakte Dokumentation von Anamnese, klinischen und apparativen Befunden sowie natürlich auch der Behandlung ist unerlässlich.

Gerade heutzutage, wo Ärzte und Kostenträger unter erheblichen finanziellen Zwängen stehen und immer häufiger Prozesse gegen Ärzte und Kliniken wegen (vermeintlicher) Behandlungsfehler angestrengt werden, kann eine exakte schriftliche und fotografische Dokumentation einer Handverletzung sowohl die Berechtigung einer stationären Behandlung beweisen als auch die Behandler vor eventuellen juristischen Konsequenzen schützen.

\section{Literatur}

1. Bain GI. Clinical Utilisation of Computed Tomography of the Scaphoid (Record Supplied by Publisher) Hand Surg 1999; 4: 3-9

2. Bickert B, Sauerbier M, Germann G. Die klinische Untersuchung des verletzten Handgelenks. Zentralbl Chir 1997; 11: 1010 - 1015

3. Blazar PE, Chan PS, Kneeland JB, Leatherwood D, Bozentka DJ, Kowalchick R. The Effect of Observer Experience on Magnetic Resonance Imaging Interpretation and Localization of Triangular Fibrocartilage Complex Lesions. J Hand Surg (A) 2001; 4: 742 -748

4. King GJ, McMurtry RY. Physical examination of the wrist and Hand. In: Gilula LA, Yin Y (eds): Imaging of the wrist and hand. Saunders, Philadelphia 1996

5. Teefey SA, Middleton WD, Boyer MI. Sonography of the hand and wrist. Semin Ultrasound CT MR 2000; 3: 192 -204

6. Watson HK, Ashmead D, Makhlouf MV. Examination of the scaphoid. J Hand Surg (A) 1988; 4: 657-660

7. Wittemann M, Jung A, Hornung R, Germann G. Die so genannte „kleine Handverletzung“ und ihre sozioökonomischen Folgen. Chirurg 1994; 11: 1004-1007

\section{Dr. med. Michael Wittemann \\ Oberarzt}

\section{Dr. med. Berthold Bickert}

Oberarzt

Univ.-Prof. Dr. med. Günter Germann Chefarzt

Klinik für Hand-, Plastische und Rekonstruktive Chirurgie

- Schwerbrandverletztenzentrum BG-Unfallklinik

Ludwig-Guttmann-Straße 13

67071 Ludwigshafen 